\title{
CAMARÕES DE PROFUNDIDADE COLETADOS NO NORTE DO BRASIL (CRUSTACEA: PENAEIDEA E CARIDEA)
}

\author{
Marilena RAMOS-PORTO \\ Professor Adjunto do Departamento de Pesca da UFRPE \\ Kátia Cristina de Araújo SILVA \\ Engenheira de Pesca - Cento de Pesquisa e Extensão Pesqueira do Norte do Brasil \\ (CEPNOR/IBAMA-MMA) \\ Girlene Fábia Segundo VIANA \\ Bióloga - Departamento de Pesca da UFRPE \\ Israel Hidenburgo A. CINTRA \\ Engenheiro de Pesca - Cento de Pesquisa e Extensão Pesqueira do Norte do Brasil
} (CEPNOR/IBAMA - MMA)

\section{RESUMO}

A realização de prospecções entre as isóbatas de 300 e 600 metros, na Região Norte brasileira, entre os Estados do Amapá e Maranhão, a partir da implantação do Programa "REVIZEE", propiciou a obtenção de um excelente acervo zoológico. Entre estes, ressaltam-se os crustáceos, representados, primordialmente, pelos Penaeidea e Caridea. As coletas foram realizadas durante o segundo semestre de 1996 e primeiro de 1997 e 1998, a bordo do Navio de Pesquisa "Almirante Paulo Moreira", sob a responsabilidade do Centro de Pesquisa e Extensão Pesqueira do Norte do Brasil (CEPNOR/IBAMA-MMA), utilizando-se redes de arrasto de fundo, objetivando-se, além do conhecimento da fauna da Zona Econômica Exclusiva do Brasil (ZEE), verificar a ocorrência, em águas de maior profundidade, de espécies potencialmente econômicas. Foram identificadas 14 espécies, distribuídas em 11 gêneros e 7 famílias. Quanto à profundidade, a faixa compreendida entre as isóbatas de 300 e $400 \mathrm{~m}$ foi a mais rica em número de espécies. Por outro lado, na faixa entre 400 e $600 \mathrm{~m}$ foram coletadas as espécies consideradas como de maior importância potencial, merecendo um estudo mais acurado Aristeus antillensis A. Milne Edwards \& Bouvier, 1909 e Aristaeopsis edwardsiana (Johnson, 1867). Para Heterocarpus ensifer A. Milne Edwards,1881, que ocorreu entre 300 e 400m, também são válidas estas recomendações.

Palavras-chave: Camarões de águas profundas; Crustacea Decapoda, Programa REVIZEE.

\section{ABSTRAT \\ Deep Shrimps from North Brazil (Crustacea: Penaeidea and Caridea)}

Prospections made between 300 and 600 meters depth off Northern Brazil from Amapá to Maranhão States, as part of the REVIZEE Program, have provided a very important zoological collection of different organisms. Among them, outrank the crustaceans, mainly Penaeidea and Caridea. Samples were made on the second semester of 1996 and first semesters of 1997 and 1998, by R/V "Almirante Paulo Moreira", under supervision of the Centro de Pesquisa e Extensão Pesqueira do Norte do Brasil (CEPNOR/IBAMA-MMA). The sampler was an otter trawl and objectives were to 
identify the fauna in the Brazilian Exclusive Zone (ZEE), and to search for species with any possible economic value. Fourteen species from eleven one genera and seven families were identified. The richest depth on number of species was between 300 and 400 meters. However, species with economic importance and that need detailed studies such as Aristeus antillensis A. Milne Edwards \& Bouvier, 1909 and Aristaeopsis edwardsiana (Johnson, 1867) were found on the depths between 400 and 600 meters. Heterocarpus ensifer A. Milne Edwards,1881, which occurred between 300 and 400 meters, also requires further studies as a potential specie of economic value.

Key-words: Deep water shrimps; Decapod Crustacea; REVIZEE Program

\section{INTRODUÇÃO}

A fauna de camarões de águas profundas do litoral brasileiro é relativamente pouco conhecida, à exceção de alguns registros, como por exemplo, os relatados por Bate (1888), a partir dos estudos dos crustáceos coletados durante e Expedição "Challenger"; por Moreira (1901) que refere espécies mencionadas por Bate (op. cit.); e por Holthuis (1971), que estudou os Glyphocrangonidae do Atlântico Ocidental. Mais recentemente, D'Incao (1995), relaciona algumas espécies coletadas em águas profundas do Atlântico Sul Ocidental.

Além destes, existem os trabalhos de Ramos-Porto, Silva \& Cintra (1997a; 1997b); Ramos-Porto, Silva, Viana \& Cintra (1998a ,1998b,1998c); Silva, RamosPorto \& Cintra (1997); Silva, Ramos-Porto, Viana \& Cintra (1997), que publicaram, de forma preliminar, informações sobre algumas espécies coletadas na região Norte do Brasil.

Por outro lado, oportunidades de pesquisas sobre esta fauna são muito raras, pois implicam, diretamente, em realizações de expedições oceanográficas em águas além da plataforma continental, as quais são, geralmente, limitadas por seu alto custo e tecnologia de coleta.

A necessidade do conhecimento da fauna habitante da Zona Econômica Exclusiva (ZEE), do Brasil, todavia, levou à implementação do Programa REVIZEE, de fundamental importância político-econômica e estratégica para o Brasil, pois tem como objetivo o levantamento do potencial dos recursos vivos desta zona, o que permitirá assegurar a sua ocupação e uso.

Neste programa, realizado com o apoio da Marinha do Brasil e de várias Instituições Federais de Ensino ou de Pesquisa, estão sendo desenvolvidos, além da identificação da fauna e trabalhos sobre sua dinâmica, investigações sobre espécies com potencial econômico .

Assim sendo, o Centro de Pesquisa e Extensão Pesqueira do Norte do Brasil (CEPNOR/IBAMA-MMA), responsável pelo REVIZEE Norte, que abrange desde a fronteira marítima com a Guiana Francesa até a foz do Rio Parnaíba, dentro de sua estratégia de trabalho, intensificou coletas entre as isóbatas de 200 e 600 metros o que propiciou a obtenção de um excelente acervo zoológico. Entre estes, os Crustacea Decapoda, grupo de reconhecida importância comercial, que congrega, além de outras, todas as espécies de camarões.

Desta forma, apresenta-se, no presente momento, uma análise sobre a composição da fauna e distribuição batimétrica dos camarões Penaeidea e Caridea coletados, procurando-se verificar, também, a ocorrência de espécies de importância 72 
econômica potencial, e em que profundidades estas ocorrem, ficando para outra oportunidade as informações sobre a dinâmica destes animais.

\section{MATERIAL E MÉTODOS}

Foram realizados 12 cruzeiros de prospecções pesqueiras para recursos demersais, ao longo da área definida para o REVIZEE Norte, executados entre os estados do Amapá e do Maranhão, pelo Navio de Pesquisa "Almirante Paulo Moreira", utilizando-se, como petrecho de pesca, uma rede de arrasto comercial para camarões; os espécimes foram coletados durante o segundo semestre de 1996 e primeiro de 1997 e 1998.

Posteriormente, estes foram acondicionados em recipientes etiquetados, anotando-se as seguintes informações: prospecção, data, posição, profundidade e tipo de substrato; após o desembarque os camarões foram estocados em câmara frigorífica $\left(-30^{0} \mathrm{C}\right)$.

Todo o material estudado está conservado em álcool a 75\%, estando depositado nas coleções carcinológicas dos Laboratórios do CEPNOR e do Departamento de Pesca da Universidade Federal Rural de Pernambuco (UFRPE).

As espécies foram identificadas à luz da bibliografia pertinente, citadas abaixo de cada espécie. Estão também mencionados, para cada uma, os seguintes tópicos: registros anteriores para o Brasil (sem nenhuma pretensão exaustiva); distribuição e habitat (ambos retirados da bibliografia); material examinado (incluindo prospecção; local; latitude; longitude; número de exemplares estudados, e, quando possível, o sexo); profundidade; tipo de fundo, quando determinado; comentários, quando pertinentes, e interesse na pescaria.

As famílias estão dispostas em ordem sistemática, de acordo com a classificação de Bowman \& Abele (1982) e seus gêneros e espécies, apresentados em ordem alfabética.

Para o estudo da distribuição batimétrica foram consideradas as seguintes faixas: nas proximidades da isóbata de 200; entre 300 e $400 \mathrm{~m}$ e entre 400 e 600 metros.

\section{RESULTADOS}

\section{Composição}

Foram identificadas 14 espécies (abaixo relacionadas), distribuídas em 11 gêneros e 7 famílias.

INFRAORDEM PENAEIDEA RAFINESQUE, 1815

Família Aristeidae Wood-Mason, 1891

Aristeus antillensis A . Milne Edwards \& Bouvier, 1909

(1997).

Referências .- Takeda (1983). D’Incao (1995). Pérez Farfante \& Kensley (1998a). 
Distribuição e Habitat .- Atlântico Ocidental: Delawere, Carolina do Norte, Carolina do Sul, Flórida, Louisiana, Texas, Antilhas, Suriname, Guiana Francesa, Brasil (Pará, Maranhão) (D’Incao, 1995; Ramos-Porto, Silva, Viana \& Cintra, 1998a).

As bibliografias consultadas referem Aristeus antillensis em fundos lamosos.

Material examinado .- Prospecção IV: Pará, $02^{\circ} 44^{\prime} \mathrm{N}-47^{0} 40^{\prime} \mathrm{W}, 2$ machos,

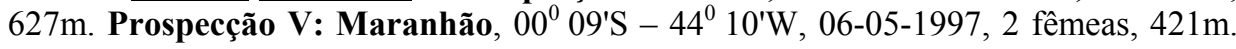
Em ambos os casos, não existem referências sobre a natureza do substrato.

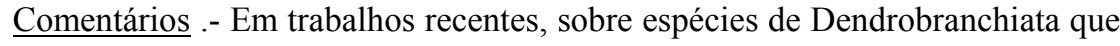
ocorrem no Brasil e Atlântico Ocidental, D’Incao (1995; 1998), não registrou Aristeus antillensis para a costa brasileira. Pérez Farfante \& Kensley (1997), que estudaram os camarões Penaeoidea e Sergestoidea do mundo, também não a mencionaram para o Brasil. Pela área de ocorrência desta espécie, contudo, era de se esperar sua coleta no setor estudado, visto que seu registro mais ao sul corresponde a Guiana Francesa, aludida por Takeda (1983).

Interesse na Pescaria .- Apesar de seu grande porte (cerca de $15 \mathrm{~cm}$ de comprimento total), não existem informações sobre o potencial pesqueiro de $A$. antillensis. Holthuis (1980), embora não a registre em seu catálogo, refere outras espécies deste gênero, ressaltando, porém, que apenas uma é pescada comercialmente, ao longo do noroeste da África, e nas costas mediterrâneas da Espanha, França, Itália e Malta. Espécie de Aristeus ocorre, consoante Holthuis (op. cit.), entre 180 e 1.440m., o que dificulta a realização de pescarias comerciais, para a maioria delas.

\author{
Aristaeopsis edwardsiana (Johnson, 1867)

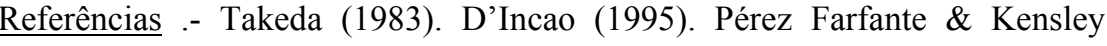

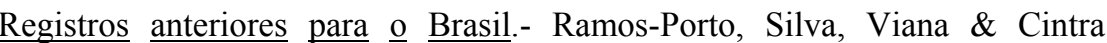
(1998a).

Distribuição e Habitat .- Atlântico Ocidental: Canadá (Terra Nova), Flórida, Antilhas, Venezuela, Guiana Inglesa, Suriname, Guiana Francesa, Brasil (Pará; Santa Catarina) e Uruguai. Atlântico Oriental. Indo-Pacífico. Ocorre entre 200 e 1850m, porém é mais comum entre 400 e 900 m (D’Incao, 1995).

Material examinado .- Prospecção IV: Costa do Pará, $02^{\circ} 44^{\prime} \mathrm{N}-47^{\circ} 39^{\prime} \mathrm{W}$, 05/10/1996, 3 exemplares, $632 \mathrm{~m}$. Sem dados sobre o tipo de fundo.

Comentários .- À exceção do trabalho de Pérez Farfante \& Kensley (1997), nas demais referências esta espécie está mencionada como Plesiopenaeus edwardsianus Johnson, 1867.

Interesse na Pescaria .- Holthuis (1980), informa que Aristaeopsis edwardsiana (citada como Plesiopenaeus edwardsianus), é pescada comercialmente em Vigo, Huelva e Cádiz (Espanha); em áreas do Senegal, Guiné, e, especialmente, no Congo e Angola $\left(4^{0}\right.$ a $\left.10^{0} \mathrm{~S}\right)$; os camarões são comercializados congelados.

Espécie de grande porte, com comprimento total máximo, já registrado, de 19 $\mathrm{cm}$ (machos) e 33,4 cm (fêmeas).

Família Penaeidae Rafinesque, 1815 

brasiliensis).

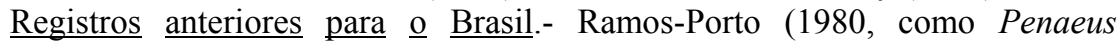

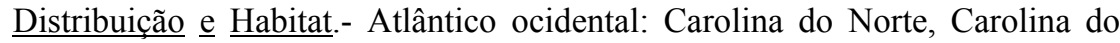
Sul, Flórida, México, Bermudas, Bahamas, Cuba, Antilhas, Nicarágua, Panamá, Colômbia, Venezuela, Guiana, Suriname, Guiana Francesa e Brasil (desde o Amapá até o Rio Grande do Sul) (D'Incao, 1995).

Material examinado - Prospecção III: Amapá, $03^{\circ} 05^{\prime} \mathrm{N}-4^{\circ} 51^{\prime} \mathrm{W}$, 15/11/1996, 8 espécimes, $348 \mathrm{~m}$. Sem dados sobre o tipo de fundo.

Comentários -- F. brasiliensis é predominantemente costeira, embora a literatura mencione sua ocorrência até 366 metros de profundidade. Em águas do Atlântico ocidental brasileiro foi coletada, principalmente, em profundidades inferiores a $60 \mathrm{~m}$, em diversos tipos de fundo, tais como areia, areia com conchas, lama, lama biodetrítica, algas calcárias, prados de Halodule, entre outros. D'Incao (1995), registroua em pradarias de Ruppia maritima, na Lagoa dos Patos.

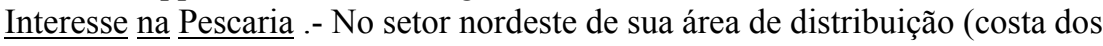
Estados Unidos; Antilhas), esta espécie geralmente representa uma pequena percentagem na captura total de camarões, porém é relativamente importante em algumas localidades do Caribe, nas Américas Central e do Sul (Quintana Roo, México), Nicarágua e Venezuela (Holthuis, 1980).

Nas Regiões Sudeste e Sul do Brasil é capturada, com maior freqüência, nas águas costeiras mais quentes, em fundos de areia/lama, com menos de 45 metros de profundidade (Paiva, 1997).

Comprimento total máximo registrado: $19,1 \mathrm{~cm}$ para os machos e $15 \mathrm{~cm}$ para as fêmeas (Holthuis, 1980).

\section{Penaeopsis serrata Bate, 1881}

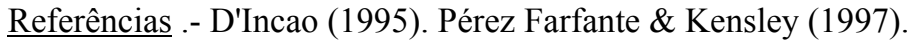

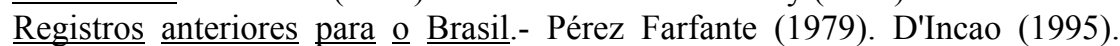
Silva, Ramos-Porto \& Cintra (1997). Ramos-Porto, Silva, Viana \& Cintra (1998c).

Distribuição geográfica.- Atlântico ocidental: Nova Jersey, Carolina do Norte, Carolina do Sul, Geórgia, Flórida, Texas, México, Bahamas, Cuba, Antilhas, Nicarágua, Panamá, Colômbia, Venezuela, Guiana, Suriname, Guiana Francesa e Brasil (Paraná; Santa Catarina; Rio Grande do Sul). Habita fundos lamacentos e arenosos, entre 183 e 750 metros de profundidade, porém concentram-se, preferencialmente, entre 300 e 450 metros (D'Incao, 1995).

Material examinado -Prospecção II: Amapá, 04º7’N - 49² $21^{\text {'W }}$, 06/10/1996, 60 exemplares, $330 \mathrm{~m}$, lama.

Comentários .- Com os dados atuais pode-se ampliar o conhecimento sobre a área de ocorrência desta espécie, e a lacuna existente, correspondendo ao setor entre a Guiana Francesa e o Paraná fica bastante reduzida, o que reforça a hipótese de que a maioria das espécies que possui repartição disjunta, com um maior esforço de coleta, deixaria de apresentar hiato em suas áreas de distribuições. 


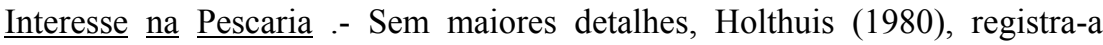
como de importância econômica potencial no Atlântico ocidental e oriental.

No Atlântico Sul ocidental, todavia, não existem dados sobre a pesca comercial de $P$. serrata. Além disso, sua profundidade de ocorrência explica o desinteresse por sua pesca, além de não haver, ainda, informações concretas sobre a dinâmica de suas populações.

Comprimento máximo registrado: $10,5 \mathrm{~cm}$ para os machos e $14 \mathrm{~cm}$ para as fêmeas (Holthuis, 1980).

Família Solenoceridae Wood-Mason, 1891

Mesopenaeus tropicalis (Bouvier, 1905)

Referências .- Pérez Farfante (1977). D'Incao (1995). Pérez Farfante \& Kensley (1997).

Registros anteriores para o Brasil.- Coelho \& Ramos-Porto (1980).

Distribuição geográfica e Habitat .- Carolina do Norte, Carolina do Sul, Geórgia, Flórida, México, Bahamas, Antilhas, Nicarágua, Panamá, Venezuela, e Brasil (Amapá; Maranhão; Espírito Santo; Rio de Janeiro; São Paulo; Rio Grande do Sul). A maioria das informações, na literatura, menciona $M$. tropicalis para profundidade entre 30 e 915 metros, embora a maior parte refira valores superiores a $100 \mathrm{~m}$ (D'Incao, 1995).

Material examinado - Prospecção IX: Costa do Pará, $01^{\circ} 29^{\prime} \mathrm{N}-46^{\circ} 43^{\prime} \mathrm{W}$, 25/05/1998, 1 macho, 6 fêmeas, 214 metros, sem informações sobre o tipo de fundo.

Comentários .- Iwai (1973, apud D'Incao 1995), registrou-a, nas Regiões Sudeste e Sul do Brasil, em fundos de calcário e cascalho; no entanto, o material estudado por este último autor foi coletado em substratos de areia e lama.

Apesar dos dados da amostra analisada não mencionarem o tipo de fundo, pela área de coleta e profundidade este é, provavelmente, lamoso.

Pérez Farfante (1977), também registrou a ocorrência de M. tropicalis em águas brasileiras, a partir de espécimes coletados no Amapá (Foz do Rio Amazonas) e Maranhão, durante a Expedição "Oregon", e em São Paulo, estes últimos provenientes das capturas realizadas durante as prospecções dos navios oceanográficos "Prof. W. Besnard" e "Calypso".

Interesse na Pescaria .- Holthuis (1980), não relaciona nenhuma espécie de Mesopenaeus como de importância comercial.

Comprimento total máximo registrado: machos (2,5cm de carapaça); fêmeas (2,8 cm de carapaça) (Pérez Farfante, 1977).

INFRAORDEM CARIDEA DANA, 1852

Família Oplophoridae Dana, 1852

Acanthephyra eximia Smith, 1884 
Referências .- Crosnier \& Forest (1973). Chace (1986).

Registros anteriores para o Brasil .- Bate (1888). Moreira (1901) (ambos como A. edwardsii). Coelho \& Ramos (1972).

Distribuição geográfica.- Atlântico ocidental: leste dos Estados Unidos, Bermudas, Bahamas, Golfo do México, Brasil (Aracajú). Atlântico oriental. Mediterrâneo. Indo-Pacífico (Crosnier \& Forest, 1973. Chace, 1986).

Material examinado - Prospecção IV: Costa do Pará, $02^{\circ} 42 \mathrm{~N}-49^{\circ} 05^{\prime} \mathrm{W}$, 07/12/1996, 2 exemplares; sem informações sobre a profundidade de captura.

Comentários .- Bate (1888), referiu $A$. edwardsii, descrita naquela ocasião e atualmente sinônima de $A$. eximia, como coletada ao sul de Pernambuco, durante a passagem da expedição "Challenger" às águas brasileiras. Apesar de estarem aludidas as coordenadas geográficas $\left(10^{0} 46^{\prime} \mathrm{S}-36^{\circ} 08 \mathrm{~W}\right)$, e o número da estação de coleta (126), autores subseqüentes, como por exemplo Moreira (1901), assinalaram Pernambuco como local da captura desta espécie. Coelho \& Ramos (1972) e Ramos-Porto \& Coelho (1998), por outro lado, mencionaram Alagoas.

Pela observação das coordenadas, no entanto, pode-se verificar que houve um engano, e que esta espécie foi capturada, na realidade, ao largo de Aracajú, Sergipe. Holthuis (1980)

Interesse na Pescaria .- Nenhuma espécie, deste gênero, é mencionada por

\section{Oplophorus gracilirostris A. Milne Edwards, 1881}

Referências .- Takeda (1983). Chace (1986).

c).

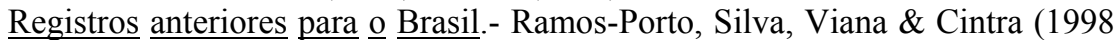

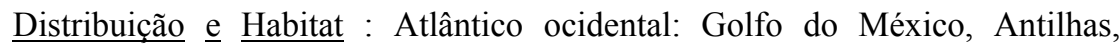
Bahamas, Brasil (Amapá; Pará). Atlântico oriental. Indo-Pacífico. Habitam profundidades entre 100 e $2.400 \mathrm{~m}$ (Takeda, 1983).

Material examinado .- Prospecção III: Amapá, $03^{\circ} 05^{\prime} \mathrm{N}$ - $43^{\circ} 04^{\prime} \mathrm{W}$, 19/11/1996, 2 espécimes, 348 m. Prospecção IV: Pará, 0240’N - 4744’W, 15/11/1996, 2 espécimes, 453m.

Interesse na Pescaria .- Holthuis (1980), não registra nenhuma espécie deste gênero como de importância comercial.

Família Psalidopodidae Wood Mason \& Alcock, 1892

Psalidopus barbouri Chace, 1939

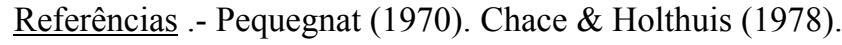

b).

Registros anteriores para o Brasil.- Ramos-Porto, Silva, Viana \& Cintra (1998

Distribuição e Habitat .- Atlântico Ocidental: talude continental da costa leste da Flórida, do Golfo do México, da Venezuela e do Suriname, bem como os taludes insulares das ilhas do Caribe (principalmente Belize) e Brasil (Pará) (Chace \& Holthuis, 1978. Ramos-Porto, Silva, Viana \& Cintra, 1998b). Oceano Índico. IndoPacífico (Pequegnat, 1970). 
Material examinado .- Prospecção III: Pará, $02^{\circ} 55^{\prime} \mathrm{N}-47^{\circ} 53^{\prime} \mathrm{W}, 12 / 11 / 1996$,

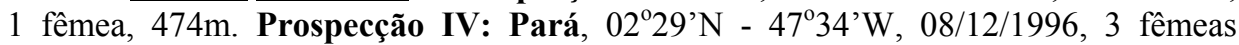
ovadas, $436 \mathrm{~m}$.

Comentários .- Segundo Thompson (1963, apud Chace \& Holthuis, 1978), a citação desta espécie para o Suriname, coletada durante a Expedição "Oregon", é questionável, porém Bullis \& Thompson (1965), não demonstraram dúvidas quanto à posição oceanográfica daquela estação.

Poucos são os registros de $P$. barbouri na literatura, estando ligados, primordialmente, a amostras coletadas durante expedições oceanográficas que exploraram as águas profundas da Região Atlântica Ocidental e Indo-Pacífica.

Interesse na Pescaria .- Holthuis (1980), não menciona nenhuma espécie deste gênero como de importância comercial.

Apesar do tamanho relativamente grande, em torno de $12-13 \mathrm{~cm}$ de comprimento total, a espécie não oferece nenhum atrativo como alimento, o que pode ser explicado pela sua aparência (corpo totalmente revestido por espinhos), e pouca carne para consumo.

Família Pandalidae Haworth, 1825

\section{Heterocarpus ensifer A. Milne Edwards, 1881}

Referências .- Pequegnat (1970). Crosnier \& Forest (1973).

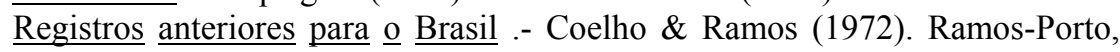
Silva, Viana \& Cintra (1998 c).

Distribuição e Habitat : Atlântico Ocidental: Carolina do Norte, Antilhas, Golfo do México, Brasil (Amapá, Pará, Maranhão). Atlântico Oriental (Crosnier \& Forest, 1969). Segundo Holthuis (1980), a espécie distribui-se entre 146 até 885 m, em fundo lamoso.

Material examinado .- Prospecção II: Amapá, $04^{\circ} 07^{\prime} \mathrm{N}$ - $49^{\circ} 21^{\prime} \mathrm{W}$, 06/10/1996, 10 exemplares, $330 \mathrm{~m}$, lama; 04º8' $\mathrm{N}-4^{\circ} 29^{\circ} \mathrm{W}, 06 / 10 / 1996,9$ espécimes, 398 m, lama. Prospecção III: Amapá, 03ํ50'N - 48 $51^{\text {' }} \mathrm{W}, 15 / 11 / 1996,125$

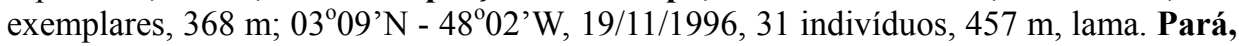
$02^{\circ} 55^{\prime} \mathrm{N}-47^{\circ} 53^{\prime} \mathrm{W}, 19 / 11 / 1996,18$ exemplares, $474 \mathrm{~m}$, lama. Prospecção IV: Pará, $02^{\circ} 40^{\prime} \mathrm{N}-47^{\circ} 44^{\prime} \mathrm{W}, 07 / 12 / 1996,1.474$ exemplares, $415 \mathrm{~m}$, lama; 02 ${ }^{\circ} 29^{\prime} \mathrm{N}-47^{\circ} 34^{\prime} \mathrm{W}$, 08/12/1996, 329 espécimes, $425 \mathrm{~m}$, lama. Prospecção V: Maranhão, $00^{\circ} 09^{\prime} \mathrm{N}$ $44^{\circ} 10^{\prime} \mathrm{W}, 06 / 05 / 1997,8$ exemplares, $421 \mathrm{~m}$.

Interesse na Pescaria .- Importância potencial; coletadas entre 250 e $650 \mathrm{~m}$ de profundidade, com armadilhas para peixes, próximo a Madagascar, Reunion e Havaí (Holthuis, 1980).

Plesionika acanthonotus (Smith, 1882)

Referências .- Pequegnat (1970). 


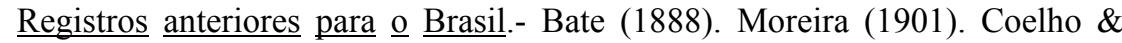
Ramos (1972). Ramos-Porto, Silva, \& Cintra (1997 b). Ramos-Porto, Silva, Viana \& Cintra (1998 c). Ramos-Porto \& Coelho (1998).

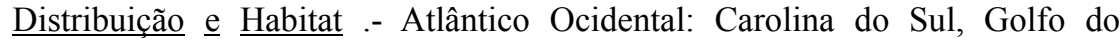
México Antilhas, Brasil (Amapá, Pará, Maranhão, Alagoas). Atlântico Oriental. Ocorre entre 330 e $1.350 \mathrm{~m}$ de profundidade (Takeda, 1983). Holthuis (1980), menciona como profundidade mínima de ocorrência 190m, em fundos de lama.

Material examinado .- Prospecção II: Amapá, 04\%07'N - 49 $21^{\circ} \mathrm{W}$, 06/10/1996, 49 indivíduos, $330 \mathrm{~m}$. Prospecção III: Amapá, 03 ${ }^{\circ} 50$ ’ N - 48 $51^{\circ} \mathrm{W}$, 15/11/1996, 10 exemplares, $368 \mathrm{~m}$, quebra do talude. Prospecção IV: Pará, $02^{\circ} 40^{\prime} \mathrm{N}$ -

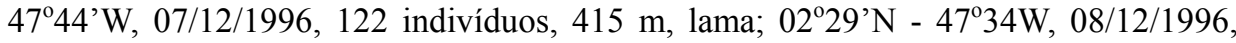

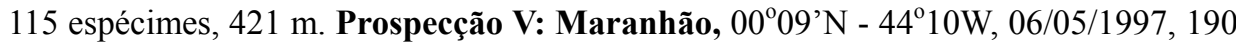
exemplares, $421 \mathrm{~m}$.

Comentários .- Os registros mais antigos desta espécie, para o Brasil, devem-se a Bate (1888) e Moreira (1901), que a mencionaram para Alagoas, ambos como Nothocaris geniculatus.

Interesse na Pescaria .- Pequeno. Zariquiey Alvarez (1946, apud Holthuis, 1980), mencionou-a como capturada na costa catalã da Espanha, porém participando com uma pequena percentagem nas pescarias. Ainda de acordo com Holthuis (op. cit.), Massuti (1968), também a registrou como de interesse comercial pequeno ou nulo.

Comprimento total máximo $8,4 \mathrm{~cm}$.

\section{Plesionika ensis (A. Milne Edwards, 1881)}

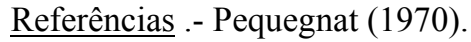

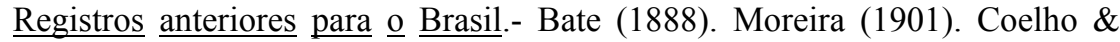
Ramos (1972). Ramos-Porto \& Coelho (1998). Ramos-Porto, Silva, Viana \& Cintra (1998 c).

Distribuição e Habitat .- Atlântico Ocidental: Flórida, Golfo do México, Antilhas, Guianas, Brasil (Maranhão; Alagoas). Atlântico Oriental. Oceano Índico. Indo-Pacífico. (Pequegnat, 1970). Registrados para profundidades entre 100 e $1.250 \mathrm{~m}$, em fundos de lama (Holthuis, 1980).

Material examinado.- Prospeç̧ão V: Costa do Maranhão, $00^{\circ} 09^{\prime} \mathrm{N}$ 44 ${ }^{\circ} 10^{\prime} \mathrm{W}, 06 / 05 / 1997,6$ indivíduos, $421 \mathrm{~m}$.

Comentários .- As menções mais antigas desta espécie, para o Brasil, devem-se a Bate (1888) e Moreira (1901), que a registraram para Alagoas, ambas como Plesionika uniproducta (em parte, apenas o abdome).

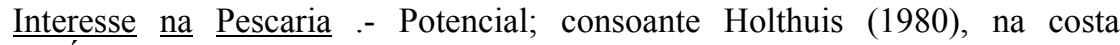
ocidental da Índia.

Tamanho máximo registrado: $18,8 \mathrm{~cm}$.

Plesionika martia (A. Milne Edwards, 1883)

Referências .- Pequegnat (1970). 


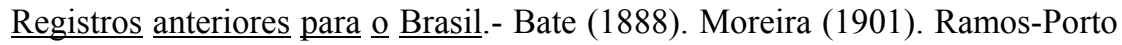
\& Coelho (1998). Ramos-Porto, Silva, Viana \& Cintra (1998 c).

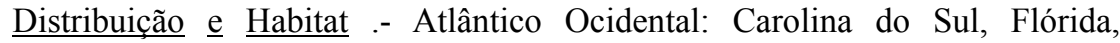
Bermudas, Golfo do México, Brasil (Pará; Alagoas). Atlântico Oriental. Mediterrâneo. Indo-Pacífico. Registrados em fundos de lama, entre 180 e $2.1 .00 \mathrm{~m}$ de profundidade (Pequegnat, 1970. Holthuis, 1980).

Material examinado .- Prospecção III: Pará, 025'N $-47^{\circ} 53^{\prime} \mathrm{W}, 19 / 11 / 1996$, 61 exemplares, 474 m, lama. Prospecção IV: Pará, 02 ${ }^{\circ} 29^{\prime} \mathrm{N}-47^{\circ} 34^{\prime} \mathrm{W}, 08 / 12 / 1996$, 120 indivíduos, $425 \mathrm{~m}$, lama.

Comentários .- As menções mais antigas desta espécie, para o Brasil, devem-se a Bate (1888) e Moreira (1901), que a registraram para Alagoas, ambas como Plesionika uniproducta (em parte, apenas a carapaça).

Interesse na Pescaria .- Potencial; pescada ocasionalmente em alguns setores de sua área de ocorrência, como, por exemplo, na costa ocidental da Índia (Holthuis, 1980). Crosnier \& Jouannic (1973, apud Holthuis, 1980), registraram-na entre as espécies "éventuellement commercialisables", no talude continental de Madagascar.

Tamanho máximo registrado: $16,9 \mathrm{~cm}$.

Família Glyphocrangonidae Smith, 1825

Holthuis (1980), não relata nenhuma espécie desta família como de importância comercial.

\section{Glyphocrangon neglecta Faxon, 1895}

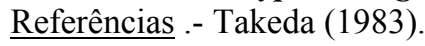

c).

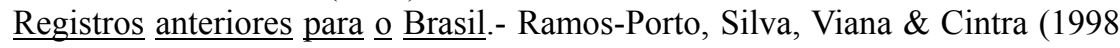

Distribuição e Habitat .- Atlântico Ocidental: Mar do Caribe, Suriname, Brasil (Amapá). Entre 365 a $1.050 \mathrm{~m}$ de profundidade (Takeda, 1983).

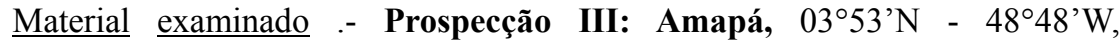
15/11/1996, 48 espécimes, $465 \mathrm{~m}$.

Comentários .- G. neglecta é de pequeno tamanho (cerca de $5 \mathrm{~cm}$ de comprimento total), não oferecendo nenhum atrativo como alimento.

\section{Glyphocrangon spinicauda (A. Milne Edwards, 1881)}

Referências .- Pequegnat (1970).

Registros anteriores para o Brasil.- Holthuis (1971). Coelho \& Ramos (1972). Ramos-Porto \& Coelho (1998). Ramos-Porto, Silva, \& Cintra (1997a). Ramos-Porto, Silva, Viana \& Cintra (1998c).

Distribuição e Habitat.- Atlântico Ocidental: costa leste da Flórida, Antilhas, Golfo do México, Yucatan, Honduras, Nicarágua, Brasil (Amapá, Pará, Maranhão). Profundidade variando entre 256 e 692 metros (Pequegnat, 1970; Holthuis, 1980).

Material examinado.- Prospecção II: Amapá, 0407'N - 49² $21^{\prime} \mathrm{W}, 06 / 10 / 1996$, 625 exemplares, $330 \mathrm{~m}$, areia. Amapá, $03^{\circ} 50^{\prime} \mathrm{N}-48^{\circ} 51^{\text {'W }}, 15 / 11 / 1996,899$ indivíduos, $368 \mathrm{~m}$, talude; $03^{\circ} 09^{\prime} \mathrm{N}-48^{\circ} 02^{\prime} \mathrm{W}, 19 / 11 / 1996,99$ indivíduos, $457 \mathrm{~m}$, lama; $03^{\circ} 09^{\prime} \mathrm{N}$ $48^{\circ} 07^{\prime} \mathrm{W}, 19 / 11 / 1996,339$ exemplares, 356 m, talude. Prospecção IV: Pará, 02 $40^{\circ} \mathrm{N}$ - 


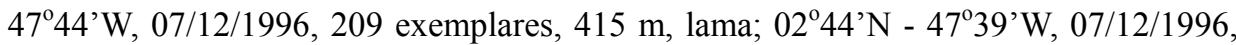

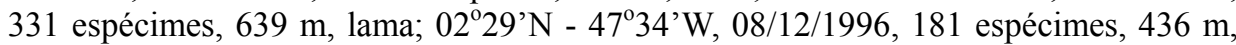

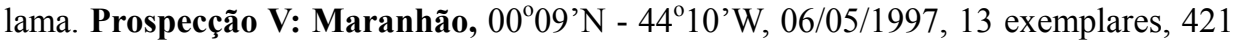
m.

Comentários .- G. spinicauda é pequena (entre 9 e $10 \mathrm{~cm}$ de comprimento total), aparentemente com pouca carne. Muito embora tenha sido capturada em quantidade relativamente grande, acredita-se que não haja interesse em sua pesca comercial, fato agravado em função de sua profundidade de ocorrência, e pelo tamanho relativo do abdome, em comparação ao cefalotórax.

\section{Distribuição batimétrica}

O setor compreendido entre $300 \mathrm{e} 400 \mathrm{~m}$ foi o mais rico em número de espécies, com uma maior ocorrência das pertencentes a Infraordem Caridea. Entre estas profundidades ocorreram Farfantepenaeus brasiliensis, Penaeopsis serrata, Oplophorus gracillirostris, Psalidopus barbouri, Heterocarpus ensifer, Plesionika acanthonotus, P. ensis, P. martia e Glyphocrangon neglecta. À exceção de $F$. brasiliensis, que ocorre preferencialmente em águas rasas, todas as demais são de ambiente mais profundo, algumas só recentemente registradas para o Brasil.

Entre 400 e $600 \mathrm{~m}$ houve um acentuado decréscimo, assinalando-se apenas 3 espécies: Aristeus antillensis, Aristaeopsis edwardsiana e Glyphocrangon spinicauda.

De forma isolada, apenas Mesopenaeus tropicalis ocorreu em torno da isóbata de $200 \mathrm{~m}$.

\section{COMENTÁRIOS E CONCLUSÕES}

Entendem-se como recursos pesqueiros todos os seres que utilizam a água como seu normal ou mais freqüente meio de vida, juntamente com um interesse econômico definido, enquadrando-se, desta forma, na categoria dos recursos naturais renováveis. Quando tais formas vivas passam a ser objeto de explotação econômica, também assumem a condição de recursos pesqueiros. Estes podem ser considerados como potenciais, quando ainda não são pescados na totalidade, ou em parte de suas áreas de ocorrência. A pesca, ao se estabelecer, tem como conseqüência direta a existência de recursos pesqueiros em explotação (Paiva, 1997).

Em sua grande maioria, as formas vivas aquáticas não apresentam qualquer valor econômico, vislumbrado em longo prazo. Estas, todavia, ocupam posições importantes em suas biocenoses, tendo em vista que nada é inútil na natureza (Paiva, 1997).

Partindo-se destes princípios, pode-se constatar, no que diz respeito às espécies ora inventariadas, que algumas atendem as qualificações mencionadas por Paiva (op. cit.), podendo ser consideradas como de importância econômica potencial.

No que diz respeito à parte dos objetivos propostos no presente trabalho, ressalta-se que, se a maioria das espécies (nove), foi coligida na faixa 300/400m, foi devido, provavelmente, à maior intensidade de coletas neste setor. 
Nestas profundidades também ocorreram camarões de valor potencial, como é o caso, por exemplo, dos pertencentes aos gêneros Plesionika, Penaeopsis e Heterocarpus.

As espécies de Plesionika, no entanto, não ostentavam uma aparência saudável para a comercialização; quando chegaram à superfície geralmente estavam com parte do estômago e dos apêndices bucais extravasados, devido às mudanças de pressão, além de exibirem os pereiópodos danificados, fruto de sua fragilidade.

Penaeopsis serrata quiçá mereça uma observação mais acurada, mas acreditase não haver um maior proveito em sua captura, pois seu tamanho máximo $(10,5 \mathrm{~cm}$, machos, e $14 \mathrm{~cm}$, fêmeas), aliado à profundidade de sua ocorrência, pode não estimular o investimento de sua captura.

Heterocarpus ensifer, coletada em número relativamente grande, talvez apresente um maior interesse, haja vista que, em outras localidades, uma espécie congênere, Heterocarpus reedi Bahamonde, 1955, foi qualificada por Holthuis (1980), como de importância considerável nas pescarias comerciais (cerca de 95\% da captura total de camarões), do Chile e do Peru. Esta espécie ocorre entre 155 e $424 \mathrm{~m}$ de profundidade, máxima, no entanto, bastante inferior a já registrada na literatura para $H$. ensifer. Salienta-se, no entanto, que os exemplares estudados no presente trabalho foram capturados em torno destas isóbatas. Lembra-se, também, que devem ser procuradas informações mais recentes nos anuários de pesca daqueles países, a fim de se verificar a atualidade destes dados, não esquecendo que, apesar de sua importância, o catálogo de Holthuis foi publicado em 1980.

Outra espécie a destacar é Farfantepenaeus brasiliensis, que, por sua vez, já participa nas pescarias da plataforma continental brasileira, embora, na Região Norte, uma outra espécie, F. subtilis (Pérez Farfante, 1967), que não ocorreu nas profundidades amostradas, seja a de maior importância comercial. Espelhando-se nas informações contidas na literatura, que mencionam a faixa entre 45 e $65 \mathrm{~m}$ de profundidade como a de maior ocorrência de $F$. brasiliensis, seu atual registro, a $368 \mathrm{~m}$, pode ser considerado esporádico.

$\mathrm{Na}$ faixa compreendida entre 400 e $600 \mathrm{~m}$ foram capturadas três espécies. Entre estas, o "camarão vermelho", Aristaeopsis edwardsiana, que, de todas, é a de maior importância potencial, devido, principalmente, ao seu grande porte. Este camarão é pescado em outros países, mas, nas coletas atuais, foi representado por poucos indivíduos. Neste setor também foi coletada Aristeus antillensis, também de tamanho comercial, que, à semelhança da precedente, ocorreu em pequena quantidade.

As demais espécies não demonstraram atrativos para a pesca comercial, conforme já foi mencionado nos comentários pertinentes.

Finalizando, sugere-se uma avaliação mais aprofundada do potencial pesqueiro e da dinâmica da população destas espécies, além da verificação da viabilidade de suas explotações, como um recurso vivo, na Zona Econômica Exclusiva (ZEE) do Mar Territorial Brasileiro. 


\section{AGRADECIMENTOS}

Ao oceanógrafo Getúlio Rincón Filho, pela confecção do Abstract. Ao CEPNOR, por colocar a disposição a coleção estudada; ao CNPq pela concessão de bolsas de pesquisas.

\section{REFERÊNCIAS BIBLIOGRÁFICAS}

BATE, C. S. , 1888. Report on the Crustacea Macrura collected by H. M. S. Challenger during the years 1873-1876. In: THE VOYAGE OF H. M. S. CHALLERGER. Zoology ... v. 29, Text. New York, Johnson Reprint Corporation, 1965. p. 1-942. Reimpressão da ed. 1888.

BOWMANN, T. E. \& ABELE, L. G., 1982. Classification of the recent Crustacea. In: ABELE, L. G. (Ed.) The Biology of Crustacea. Systematics, the fossil record, and biogeography. New York, Academic Press, v.1, cap.1, p. 1-25.

BULLIS, H. R. Jr. \& THOMPSON, J. R. , 1965. Collections by the exploratory fishing vessels Oregon, Silver Bay, Combat, and Pelican made during 1956-1960 in the southwestern North Atlantic. U. S. Fish and Wildlife Service, Special Scientific Report-Fisheries, Washington, v. 510, p. 1-130.

CHACE JR , F.A. , 1986. The caridean shrimps (Crustacea: Decapoda) of the Albatross Phyllipine Expedition, 1907-1910, Part 4: families Oplophoridae and Nematocarcinidae. Smithsonian Contribution to Zoology, Washington, n.. 432, p. $1-82$.

CHACE JR., F.A. \& HOLTHUIS, L. B. , 1978. Psalidopus : The scissors-foot shrimp (Crustacea: Decapoda: Caridea).Smithsonian Contribution to Zoology, Washington, n. 277 , p.1-22.

COELHO, P. A. \& RAMOS, M.A., 1972. A constituição e a distribuição da fauna de decápodos do litoral leste da América do Sul entre as latitudes de $5^{0} \mathrm{~N}$ e $39^{\circ} \mathrm{S}$. Trabalhos Oceanográficos, Universidade Federal de Pernambuco, Recife, v. 13, p. $133-236,1972$.

COELHO, P. A. \& RAMOS-PORTO, M., 1980. Crustáceos decápodos da costa do Maranhão, Brasil. Boletim do Instituto Oceanográfico, São Paulo, v. 29, n. 2, p.135138.

CROSNIER, A. \& FOREST, J., 1969. Note preliminaire sur les peneides recuillis par l' Ombango ", au large du plateau continental, du Gabon à L'Angola. (Crustacea: Decapoda: Natantia). Paris: Bulletin du Museum D'Histoire Naturelle, Paris, ser. 2, V. 41, n. 2, p. 544-554".

CROSNIER, A. \& FOREST, J. , 1973. Les crevettes profondes de l'Atlantique Oriental tropical. Faune Tropicale, Paris, v. 19, p. 1-409.

D'INCAO, F., 1995. Taxonomia, padrões distribucionais e ecológicos dos Dendrobranchiata (Crustacea : Decapoda) do Brasil e Atlântico Ocidental . Curitiba, 1995. 365 p. Tese (Doutorado em Ciência), Universidade Federal do Paraná.

D'INCAO, F., 1998. Malacostraca. Eucarida. Dendrobranchiata. In: YOUNG, P.S. (ED). Catalogue of Crustacea of Brazil. Rio de Janeiro: Museu Nacional. p. 311321. (Série Livros n.6). 
HOLTHUIS, L. B., 1971. The Atlantic shrimps of the deep-sea Genus Glyphocrangon A. Milne Edwards, 1881. Bulletin of Marine Science, Miami, v. 21, n. 1, p. 267373.

HOLTHUIS, L. B., 1980. FAO species catalogue; shrimps and prawns of the world. An annotated catalogue of species of interest to fisheries. Roma: FAO, v 1. p (FAO Fisheries Synopsis, 125).

MOREIRA, C. , 1901. Contribuição para o conhecimento da fauna brasileira. Crustáceos do Brasil. Archivos do Museu Nacional, Rio de Janeiro, v. 11, p. 1151.

PAIVA, M. P., 1997. Recursos pesqueiros estuarinos e marinhos do Brasil. Fortaleza. UFC Edições, 286p.

PEQUEGNAT, L. H. , 1970. Deep-sea caridean shrimps with descriptions of six new species. Texas $A \& M$ University Oceanographic Studies. I. Contribution on the Biology of the Gulf of Mexico, n. 4, p. 59-123.

PÉREZ FARFANTE, I. , 1969. Western Atlantic shrimps of the genus Penaeus. Fishery Bulletin, U.S. Fishery Wildlife Service, Washington, v. 67, p. 461-591.

PÉREZ FARFANTE, I. , 1977. American solenocerid shrimps of the genera Hymenopenaeus, Haliporoides, Pleoticus, Hadropenaeus new genus, and Mesopenaeus new genus. Fishery Bulletin, U.S. Fishery Wildlife Service, Washington, v. 75, n. 2, p. 261-346.

PÉREZ FARFANTE, I. , 1979. Range extension of Penaeopsis serrata (Crustacea Penaeoidea) to off New Jersey and Rio Grande do Sul. Proceedings of the Biological Society of Washington, Washington, v. 92, n. 1, p. 204-207.

PÉREZ FARFANTE, I. \& KENSLEY, B., 1997. Penaeoid and sergestoid shrimps and prawns of the world . Keys and diagnoses for the families and genera. Mémoires $d u$ Muséum National D'Histoire Naturelle, Paris, tomo 175, p. 1-233.

RAMOS-PORTO, M., 1980. Estudo ecológico da região de Itamaracá, Pernambuco, Brasil.,VII. Crustáceos Decápodos Natantes. Trabalhos Oceanográficos, Universidade Federal de Pernambuco, Recife, v. 15, p. 227-310.

RAMOS-PORTO, M. \& COELHO, P. A., 1998. Malacostraca. Eucarida. Caridea. In: YOUNG, P.S. (ED). Catalogue of Crustacea of Brazil. Rio de Janeiro: Museu Nacional. p. 325-350. (Série Livros n.6).

RAMOS-PORTO, M.; SILVA, K. C. A. \& CINTRA, I. H. A. 1997a. Registro de Glyphocrangon spinicauda A.Milne Edwards, 1881, na plataforma continental do Estado do Amapá, Brasil. In: ENCONTRO DE ZOOLOGIA DO NORDESTE, 11. Fortaleza, 1997. Resumos ... p.19.

RAMOS-PORTO, M.; SILVA, K. C. A. \& CINTRA, I. H. A. 1997 b. Ocorrência de espécies da família Pandalidae na plataforma continental Norte do Brasil. In: ENCONTRO DE ZOOLOGIA DO NORDESTE, 11. Fortaleza, 1997. Resumos ... p. 20.

RAMOS-PORTO, M.; SILVA, K. C. A.; VIANA, G.. F. S. \& CINTRA, I..H.A., 1998 a. Registro de Aristeus antillensis (A.Milne Edwards \& Bouvier, 1909) na costa Norte brasileira. (Crustacea: Decapoda: Aristeidae). In: CONGRESSO BRASILEIRO DE ZOOLOGIA, 22, 1998, Recife, Resumos ..., Recife, Sociedade Brasileira de Zoologia, 1998, p.74. 
RAMOS-PORTO, M.; SILVA. K. C. A.; VIANA, G. F. S. \& CINTRA, I.. H. A. , 1998 b. Psalidopus barbouri (Crustacea: Decapoda) em águas do litoral Norte brasileiro. In: CONGRESSO BRASILEIRO DE ZOOLOGIA, 22, 1998, Recife, Resumos ... , Recife, Sociedade Brasileira de Zoologia, 1998, p.100.

RAMOS-PORTO, M.; SILVA, K. C. A.; VIANA, G. F. S. \& CINTRA, I. H. A., 1998 c. Camarões de profundidade coletados na costa Norte do Brasil (Crustacea: Penaeidea e Caridea). In : CONGRESSO BRASILEIRO DE ZOOLOGIA, 22, 1998, Recife, Resumos ..., Recife, Sociedade Brasileira de Zoologia, 1998, p.101.

SILVA, K.C.A.; RAMOS-PORTO, M.; \& CINTRA, I. H. A., 1997b. Ocorrência de espécies de Penaeidae em águas da costa Norte do Brasil (Crustacea:Decapoda). IN: ENCONTRO DE ZOOLOGIA DO NORDESTE, 11. Fortaleza, 1997. Resumos ..., Fortaleza, 1997. P. 20.

SILVA, K. C. A.; RAMOS-PORTO, M.; CINTRA, I. H. A. \& VIANA, G. F. S., 1997 a. Ocorrência de Plesiopenaeus edwardsianus (Johnson, 1867) na costa Norte do Brasil (Crustacea: Decapoda: Penaeidea) In: CONGRESSO LATINOAMERICANO SOBRE CIÊNCIAS DO MAR, 7, 1997. São Paulo, Resumos Expandidos, São Paulo, Instituto Oceanográfico da Universidade de São Paulo e Associação Latino-Americana de Investigaciones em Ciências do Mar, v. 2, p. 452.

TAKEDA, M. 1983. Crustaceans and mollusks trawled off Suriname and French Guiana. Tóquio: Japan Marine Fishery Resoure Research Center, 354 p. 